\title{
Total Synthesis of (+)-Flavisiamine F
}

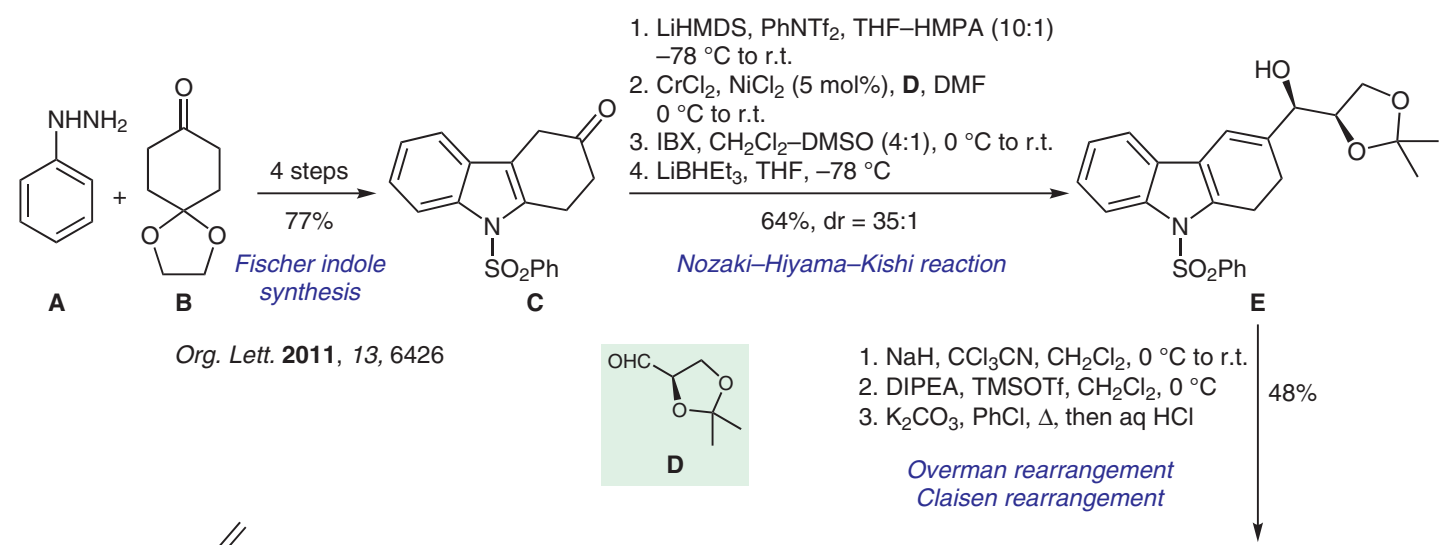<smiles>C=CCNC1c2c(n(-c3ccccc3)c3ccccc23)CC[C@@]1(C/C=C/COC)CC(=O)OC</smiles>

$\mathrm{Ag}_{2} \mathrm{O}, \mathrm{Mel}, \mathrm{MeCN}, 35^{\circ} \mathrm{C}$

2. KHMDS, TBSCI, THF, $-78^{\circ} \mathrm{C}$

3. DIBAL-H, $\mathrm{CH}_{2} \mathrm{Cl}_{2}-\mathrm{PhMe}(2.5: 1),-78^{\circ} \mathrm{C}$

4. $\mathrm{K}_{2} \mathrm{CO}_{3}$, allyl bromide, $\mathrm{MeCN}, 0^{\circ} \mathrm{C}$ to r.t.

$\mathrm{SO}_{2} \mathrm{Ph}$

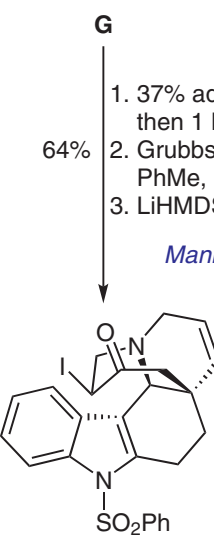

$\mathrm{H}$

a $\mathrm{HCHO}, \mathrm{EtOH}$

Mannich reaction

4. $\mathrm{K}_{2} \mathrm{CO}_{3}$, allyl bromide, $\mathrm{MeCN}, 0^{\circ} \mathrm{C}$ to r.t.
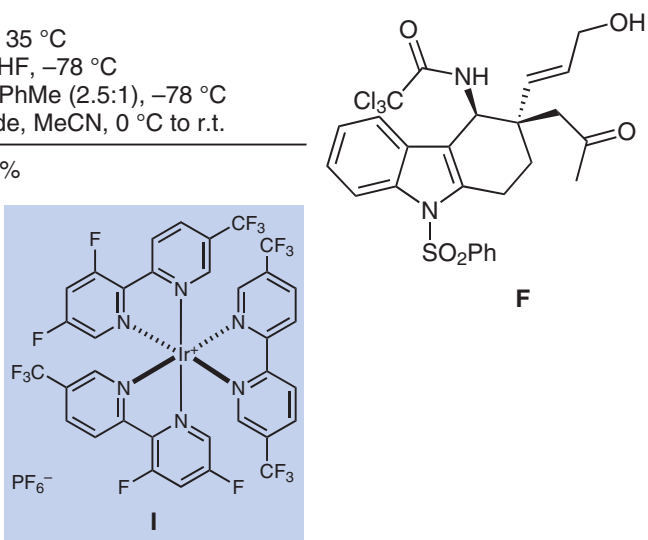

1. $\mathrm{CH}(\mathrm{CN})_{2} \mathrm{OAc}, \mathrm{Im}, \mathrm{MeOH}$

$0{ }^{\circ} \mathrm{C}$ to r.t.

2. $30 \%$ aq $\mathrm{H}_{2} \mathrm{O}_{2}, \mathrm{~K}_{2} \mathrm{CO}_{3}$

DMSO, $0^{\circ} \mathrm{C}$ to r.t.

3. $\mathrm{HCl}(\mathrm{g}), \mathrm{MeOH}, \Delta$

DMF, blue LED

$77 \%$

$38 \%$

Strecker synthesis

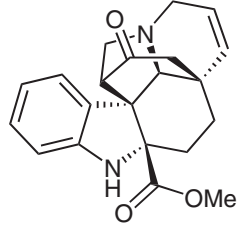

(+)-Flavisiamine $\mathrm{F}$
Significance: Xia and co-workers report the first enantioselective total synthesis of the Kopsia alkaloid (+)-flavisiamine F. The authors elegantly solve the synthetic puzzle posed by this strained polycyclic natural product by using a strategy involving two [3,3]-sigmatropic rearrangements, ring-closing metathesis, Mannich reaction, and iridiumcatalyzed radical cyclization.
Comment: Nozaki-Hiyama-Kishi coupling of the enol triflate derived from $\mathrm{C}$ followed by oxidation and stereoselective reduction gave $\mathbf{E}$. Sequential Overman and Claisen rearrangements afforded ketone $\mathbf{F}$ en route to allyl amine $\mathbf{G}$, which then underwent Mannich reaction and ring-closing metathesis. Halogenation and iridium-mediated photocyclization provided J, which was elaborated into the target molecule by Strecker reaction and hydrolysis.

\section{Key words}

(+)-flavisiamine $F$

Nozaki-HiyamaKishi reaction

Claisen

rearrangement

Overman rearrangement

photocyclization

Strecker synthesis 